\title{
GIS-based Integrated Management Platform for Electricity Payment Channels
}

\author{
NING Bo ${ }^{1}$, MA Lu Jin ${ }^{1}$, LI Ying ${ }^{1}$, QIN Hua Qin ${ }^{2}$ \\ (1.State Grid JiBei ElectricPower CompanyLimited, Beijing 100053, China; 2.BeiJing \\ KeDongElectric Power Control System Co.,Ltd.,Beijing 100085, China)
}

Keywords: electricity service channels; GIS; integrated management; channel planning

\begin{abstract}
This paper analyzed the current situation of integration managementfor payment channels in Jibei Electric Power co., LTD of State Grid, and then proposed an effective solution of GIS-based payment channel integrated management platform. The design of the platformis based on the existed integrated payment access management platform that is uniformly implemented by State Grid Corporation. The platform hasadopted GIS visualization techniques, providingmanagers with vividvisual panorama of payment channels and also the platform can aid decision making on channelsplanning and customer service guiding.
\end{abstract}

\section{Introduction}

Geographic information system (GIS) is one of the most widely used visualization technologies. With a surprising development in nearly 30 years, the visualization technology has been applied in the resource investigation, environmental assessment, disaster prediction, land management, urban planning, postal service and telecommunications, transportation, military and public security, water conservancy, electric power, public facilities management, agriculture, forestry and animal husbandry, statistics, financial and almost all other areas of modern society [2]. In the power industry, GIS technology is applied to equipment management of power distribution and information visualized display [1]. Electricity payment channels as electric industry customer service carrier, has characteristics of rich information, great variance in categories, large quantity and widely distributed nodes. The information visualization displaying of electricity payment channels play a vital role in the channel distribution planning and operation management standards.

\section{The Current Situation of Integrated Management Platform for Electricity Payment Channels}

In recent years, the Jibeielectric power co., LTD. has developed more than 20 kinds of payment channelsin order to improve the convenience of electricity customers' payment method. To further improve the security and stability of the payment channels, in February of 2012, Jibei electric power company started to deploy the unified payment access platform developed by State Grid, the platform can integrate various payment channels to a unified access, support and monitoringchannel, the platform has completed and been inspected of final acceptance in April 2014. At present, the platform has a total of 5 financial payment channels, including ICBC, Agricultural Bank of China, China Construction Bank, and 3 non-financial payment channels, including postal payment and mobile phone payment, besides, 558 self-service payment terminals, 280 POS terminals and 2 electric power service provider (website 9598 and mobile marketing application). The access channels have a 4.1885 million monthly average transactions and 534 million RMB monthly average transaction amounts. The deployment of the platform on the one hand guaranteed the electric payment service quality, on the other hand laid the foundation to the full coverage of payment channels management. Through the platform, management staff of different payment channels can monitor the service status in real time. The platform also provided a strong technical support for the management and maintenance of the payment channelson a 
daily basis.

However, with the fast development of new payment methods and huge increase in the users' demanding of better and convenient services, the traditional text analysis and display of information on web application can't provideinformation in anintuitive way with multi-dimensional interface, which will greatly help the management staff in locating the problem and rapidly forming a solving plan. Therefore, a system for electricity payment channels management with GIS marketing visualization technology and based on the present unified payment access platformis in urgent need for the payment channels management staff. This new platform with GIS technology can manage the market resources visually, provide whole scale service data analysis and rapid system maintenance with the geographical information and visualized data presenting. The new platform will increase the overall payment channel efficiency and customer satisfaction.

\section{System Design}

The integrated management platform for electricity payment channels (hereafter referred to as integrated management platform) designed in this paper is based on the already deployed unified payment access platform of Jibei electric power company, and the new integrated management platform in this paper introduced the GIS technology, provided a visualized method of electric payment channel management through data sharing and business integration.

\subsection{Design Idea}

Unified payment access platform is an important part of intelligent electricity services system. The platform in this paper was based on SOA architecture design [4], with fully support of self-service terminal payment, online payment, mobile phone payment and social agent payment. The platform also provided a unified channel for electric company and financial, non-financial payment channel.A unified access, monitoring and management can be accomplished through the platform designed in this paper. In addition, the platform also will make sure the application system, like marketing business, running stablely.

GIS is a computer-based tool, it has a mature application in production management of electric distribution network [1]. GIS can analyze and process spatial information on map, then the processing of spatial informationwill integrate with general database information, the result of the above processing can provide vivid and intuitive information to customers.

\subsection{System Structure}

The structure of integrated management platform for electricity payment channels platform based on GIS is shown in figure 1.

Base on the unified payment access platform, the integrated management platform for payment channels continues to use the architecture of the unified payment access platform, with newly introduced GIS architecture, the system structure tends to be multi-layer, and layers integration. The system structure followed the rule of service hierarchy. The system consists of data processing layer, map service layer and user oriented application layer. Data processing layer extracts, integrates, transforms all kinds of data, the map service layer provides spatial data processing, the application layer relies on the GIS to display and analyze general data.

\subsection{System Modules}

The main modules of the integrated management platform including system basic module, data processing module, map service module, basic and advanced application module. 


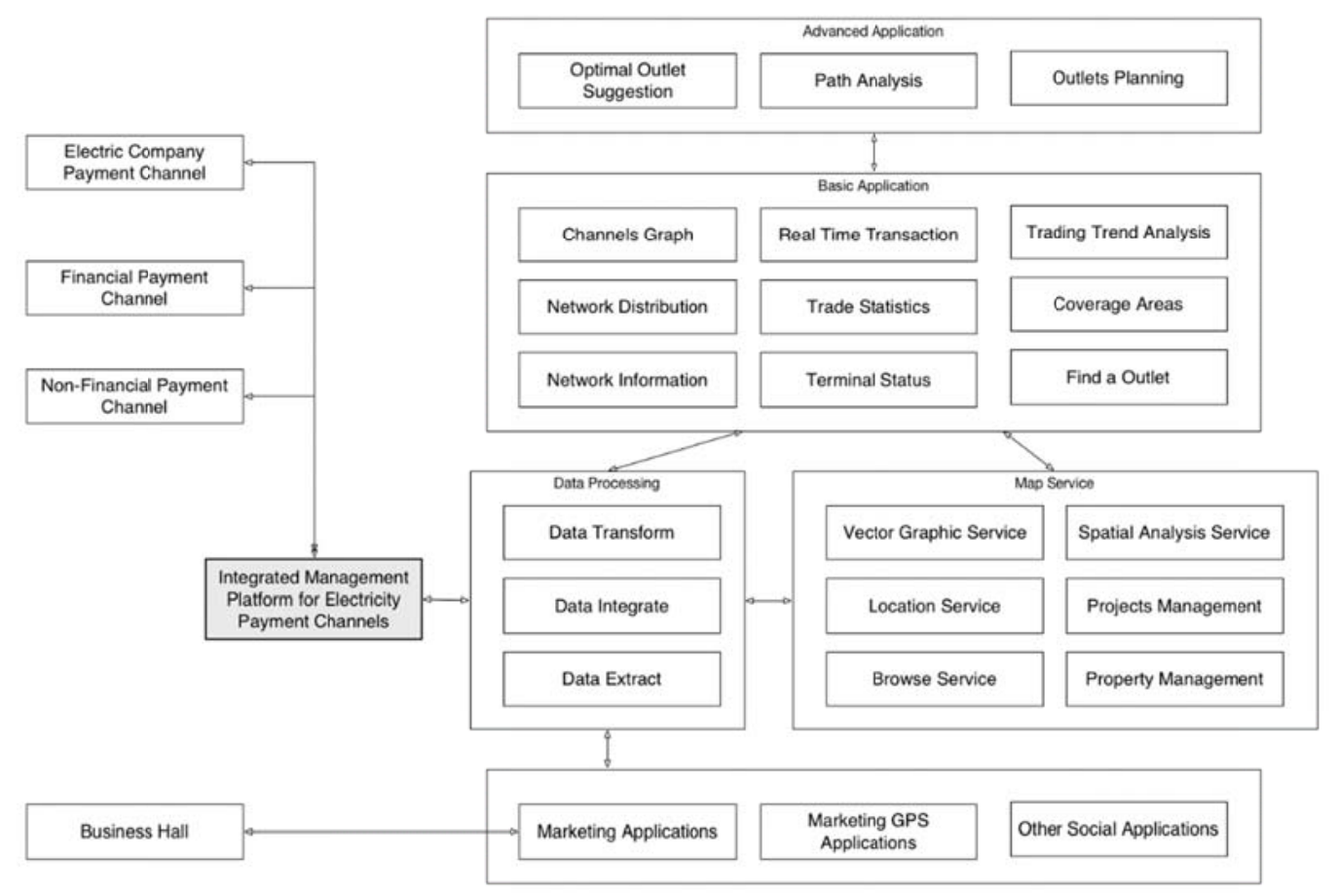

Figure 1. The structure of GIS based integrated management platform for electricity payment channels platform

\subsubsection{System basic module}

System basic module includes data access module, platform support module and map processing module. The data access module is data persistence layer, this module mainly responsible for the operation of the database and data security. Platform support module has the support module of unified payment access platform, this modulemainly provides the parameter configuration, access control, personnel organization setup and audit security functions. Map processing module providesfunction of map data processing, including map loading,basic map operations, view management and graphical output.

3.3.2 Data processing module

With OR mapping technology, the business process data and results data, spatial data can be integrated to satisfy user requirement or provide vector model data to the front-end applications. The data processing module can also load extracted data or added ports to the database, in addition, the data processing module can manage transformed data.

\subsubsection{Map service module}

Map service module mainly works with spatial data, with data processing tools, the user can create or edit the geographic information layer, dealing with the corresponding geographic data.

d. Basic application module

The module mainly provides the display of analysis result of payment data, including payment channels, network outlet information, transaction data and trend analysis, coverage area analysis and location service.

3.3.4 Advanced application module

This module mainly provides data mining and decision analysis, including: (1) electric customer oriented advanced applications, for example, the optimal network outlet suggestion and network outlet route suggestion; (2) electric payment channel management staff oriented advanced applications, for example, the channel optimization and channel development history analysis.

\subsection{Application Functions}

\subsubsection{Channels Graph}

Base on the administrative zoning map of Jibei electric power company, the channels graph supports branches view of channels. The channels graph function provides the data of channels' number, network outlets number, daily transactions and transaction amounts, local company 
proportions of transaction amounts, then the module displays the channels statistics categorized by local electric company. The channels graph function provides the overall status and information of the Jibei electric power company.

\subsubsection{Network Distribution Map}

From the channels registered information, with the geographical data of outlets, this function can display a panoramicview of the network distribution.According to the scaling of the map, the model adopts the clustering analysis method, with a certain clustering radius, the map can merge similar outlets on the map.

\subsubsection{Network Information Display}

On the network distribution map, select an outlet, the user can view the detailed information of the outlet, including outlet channel type, outlet name, outlet address, contacts, daily transactions, daily transaction amounts, online status.

\subsubsection{Real-time transactions}

According to the map information, dynamic display the power supply unit or outlets transactions within the view, including the total number of transactions, transaction amounts. At the same time, this function providesanalysis of payment and channel categories.

\subsubsection{Trading Statistics}

This function mainly works with the analysis of channels payment amount. This function also can show the statistic results of local electric power company payment data as graphs.

\subsubsection{Terminal Status}

With a panoramic view of the map, this function can show the distribution of normal operated terminals and physical location of failure terminals. The failure terminals tag with mark to alert maintenance staff to deal with the failures.

\subsubsection{Trading Trend Analysis}

This function can transverse comparison of different channels' transaction data, longitudinal comparison of transactions at different times.

\subsubsection{Coverage Areas}

According to the channel distribution outlets, this function can visually display payment network geographic area coverage. The network geographic area coverage is an intuitive basis for the new outlet location selection.

\subsubsection{Find an Outlet}

With GIS technology, this function can visually display the surrounding environment of an outlet, this function can greatly improve the customer service quality.

\subsubsection{Optimal Outlet Suggestion}

Considering of customer location, customer requirements, network outlets services capability, transportation and other factors, this function provides the customer most convenient and highest customer satisfaction outlet.

\subsubsection{Route Analysis}

According to customer's starting location and selected payment outlet, using GIS technology, this function provides users with an optimal route.

\subsubsection{Outlets Planning}

Distribution network outlets planning includes existing payment network outlets' layout optimization and newly setup outlets' location planning. Considering of urban layout, traffic planning, population distribution, supply demand and other factors, with the principles of "ten minutes' traffic circle" and "every rural area has a outlet", based on GIS technology, this function can provide decision support for the payment channel planning and service capability improvement.

\subsection{Key Information Collection}

The key information of integrated management platformincludes:outlet's spatial attributes, outlet's basic attributes, transaction operation information and channels' operation related public information.

\subsubsection{Spatial Attributes}


Spatial attributes refers to the outlets'longitude, latitude and altitude. TheGIS in this paper is limited to 2-D GIS, so the platformonly needs to collect the outlets' latitude and longitude coordinates. According to the overall informatization construction plan of state grid, the maintenance of longitude and latitude information is through the marketing GIS application. Besides, in order to avoid the impact of the out sync of project construction progress, the integrated platform provides spatial attributes manual maintenance interface and import port, and the platform also supports mobile terminal longitude and latitude data automatic gathering or manual input, and data upload.

\subsubsection{Basic Attributes}

Basic attributes refers to the network payment outlets' name, location, service time, service content, customer, supported payment method. According to the overall informatization construction plan of state grid, the maintenance of basic attributes is through the marketing business application. The integrated platform acquires basic attributes through market business application ports.

\subsubsection{Channels' Operation Information}

Channels' operation information refers to the outlets' running state (running, fault, and shutdown), transaction amounts and other operation information. According to the overall informatization construction plan of state grid, the channels' operation information is recorded by the unified payment access platform. The integrated platform accesses this data through the data sharing with unified payment access platform.

3.5.4 Public Information

Public information refers to the city planning, demographicdata, public transportation information and other public information. This kinds of information can be accessed through ports of other public service system.

\section{Conclusion}

Based on the unified payment access platform of Jibei electric power company, this paper proposed an integrated management platform for electricity payment channel. The platform proposed in this paper aim to provide visualized management methods for payment channel staff and better service guide to customers. The integrated platform can be seen as deepen application of unified payment access platform, and the integrated platform can be deployed separately or in corporation with unified payment access platform. With GIS technology, the integrated platform displayed the network outlets distribution and real-time operation in a visualized and intuitive way. Visualization management of the payment channels has achieved through the integrated platform. On the basis of present development of payment channels and outlets, the integrated platform can be deployed in the following steps: the first step is basic application implementation, a standardized management process and methods are formed in this step; the second step is integration of public information to achieve advanced application implementation, for example, the integrated platform can integrate city planning and transportation planning information to provide suggestion for the outlet setup and advanced service for customers.

\section{Reference}

[1]Yue Liguo, Zhang Guoqing. Application of geographic information system in power distribution network automation [J]. Journal of information science and technology, 2006, (33):25.

[2]Zhang Chao. Geographic information system application tutorial [M]. Beijing: science press, 2007.

[3] Yang Haiyong, Wu Jinwei, Cui Kaifu. Integrated payment access management platform application [J]. Journal of public power, 2012, (6): 20-21. 
[4] Wang Shuquan, Zou Ningfeng, Jin Xin. SOA based integrated payment management platform of research and application [J]. Journal of information technology, 2012, (10): 121-124.

[5] Wang Ling. GIS based platform for the integrated traffic information display [J]. China water transport, 2011, (12):84-85. 